\title{
Invasive pulmonary aspergillosis accompanied by soft tissue lesions during treatment of a patient with hyperthyroidism: a case report
}

\author{
XIAO-YUN FAN, WEI-MIN WANG, XUE-BO YAN, CONG-HUI WANG, RONG-YU LIU
}

Department of Pulmonology Medicine, Anhui Geriatric Institute, the First Affiliated Hospital of Anhui Medical University, Hefei 230022, Anhui, PR China

\begin{abstract}
Invasive pulmonary aspergillosis (IPA) is difficult to diagnose because it requires histopathology and tissue culture, as well as due to its rapid progression. Invasive pulmonary aspergillosis is the primary cause of pulmonary mycosis in China, which can occur in patients with neutrophil deficiency, leukaemia or lymphoma, malignant tumours, or chronic obstructive pulmonary disease with long-term corticosteroid use or bacterial exacerbations. Such fungal infections can lead to disseminated disease and death within weeks, and the mortality rate for untreated invasive aspergillosis is high. Therefore, increased awareness of invasive aspergillosis in non-traditional hosts is warranted due to the high mortality rate experienced by patients with this disease. Invasive pulmonary aspergillosis has become a principal cause of life-threatening infections in immunocompromised patients. Invasive aspergillosis frequently involves the lung parenchyma and is infrequently accompanied by soft tissue lesions. We present an unusual case of a patient with agranulocytosis that was caused by methimazole that was given to control his hyperthyroidism, and IPA that was accompanied by unusual maxillofacial soft tissue swelling that required treatment with voriconazole. Upon follow-up 11 months later, a chest computed tomography scan $(C T)$ revealed that most lesions had been completely absorbed. Moreover, his maxillofacial ulcers had become encrusted, and the soft tissue swelling had subsided.
\end{abstract}

Key words: invasive pulmonary aspergillosis, Aspergillus; voriconazole, hyperthyroidism, agranulocytosis, methimazole.

(Centr Eur J Immunol 2015; 40 (1): 117-121)

Invasive fungal infections are serious, often lethal diseases in severely immunocompromised patients [1,2]. An autopsy study concerning fungal infection has demonstrated that Candida most commonly affects the gastrointestinal tract, while Aspergillus is more likely to affect the lung [3]. Invasive pulmonary aspergillosis (IPA) is the primary cause of pulmonary mycosis in China [4]. Invasive pulmonary aspergillosis can occur in patients with neutrophil deficiency, leukaemia or lymphoma, malignant tumours, or chronic obstructive pulmonary disease (COPD) with long-term corticosteroid use or bacterial exacerbations $[2,5,6]$. Such fungal infections can lead to disseminated disease and death within weeks, and the mortality rate for untreated invasive aspergillosis is high [7, 8]. Therefore, increased awareness of invasive aspergillosis in non-traditional hosts is warranted due to the high mortality rate experienced by patients with this disease. Herein, we pres- ent a case of agranulocytosis that had invasive pulmonary aspergillosis accompanied by unusual maxillofacial soft tissue swelling.

\section{Case report}

A 43-year-old, previously healthy male was referred with complaints of recurrent fever for over 20 days and a cough with expectoration of white purulent sputum for two days before admission. He had no known history of pulmonary diseases such as COPD, asthma, underlying diseases such as diabetes, hypertension, any other diseases or risk factors of immunological deficits such as chronic diseases or recurrent infections. He had no addictions such as taking drugs, smoking, or alcoholism. Fifty days prior to referral, the patient was diagnosed with hyperthyroidism and prescribed $30 \mathrm{mg} /$ day of methimazole by an endocrinologist. The initial laboratory

Correspondence: Prof. Rong-Yu Liu, MD, PhD, Department of Pulmonology Medicine, Anhui Geriatric Institute, the First Affiliated Hospital of Anhui Medical University, Jixi Road 218, Hefei 230022, Anhui, PR China, tel. +86-551-62922807, e-mail: rongyuliu@ gmail.com 
evaluation before treatment with methimazole revealed white blood cells were $7.03 \times 10 \% / 1$ (neutrophils $56.2 \%$, lymphocytes $37.9 \%$, and eosinophils $2.3 \%$ ), haemoglobin $127 \mathrm{~g} / \mathrm{l}$, and platelets $206 \times 10^{9} / 1$. However, he did not review the blood test after taking methimazole until he exhibited a fever with a maximum temperature of $40^{\circ} \mathrm{C}$ accompanied by chills after taking methimazole for twelve days, and finished more than seven days of ineffective antibiotic treatment. With a white blood cell count of $0.7 \times 10^{9} / 1$ and absolute number of neutrophilic granulocytes $0.01 \times 10^{9} / 1$, he was on admission in the Department of Haematology, and then he ended up taking methimazole. He was then administered granulocyte colony-stimulating factor (subcutaneous injection of $450 \mu \mathrm{g} /$ day for 4 days), imipenem and cilastatin sodium, vancomycin, and caspofungin acetate for anti-infective therapy.

However, the patient's fever remained at $38^{\circ} \mathrm{C}$ and was accompanied by paroxysmal irritating cough and expectoration with white purulent sputum, and oral ulcers scattered, the skin of his left external nose and eyelid swelling, and the left nasal had acute congestion with white and yellow purulent secretions. A computed tomography (CT) of the chest revealing an air crescent sign (Fig. 1A) is highly suggestive of infection by an angioinvasive fungus, most commonly Aspergillus. The patient was transferred from the Department of Haematology to Respiratory Medicine. The patient was conscious with no enlargement of superficial lymph nodes, maxillary sinus tenderness, or maxillofacial soft tissue swelling due to inflammation (Fig. 2). On the sixth day of hospitalisation, a laboratory test showed white blood cell count of $6.27 \times 10^{9} / 1$. Aspergillus fumigatus was detected in both fungal and bacterial culture of the patient's sputum. The fungal isolate demonstrated typical characteristics of $A$. fumigatus, including colony formation, abundant sporulation, and profuse growth. The conidia were nearly spherical and appeared similar to a chrysanthemum (Fig. 3). In addition, the concentration of the PLATELIA ${ }^{\mathrm{TM}}$ ASPERGILLUS Ag (BIORAD, California, USA) was $0.5 \mathrm{pg} / \mathrm{ml}$, and the fungi (1, 3)- $\beta$-D-glucan detection system (Chinese Horseshoe Crab Reagent Manufactory Co., Ltd., Xiamen, China) detected $78.80 \mathrm{pg} / \mathrm{ml}$ of analyte. We diagnosed the patient with invasive $A$. fumigatus infection induced by agranulocytosis. He was administered voriconazole ( $0.4 \mathrm{~g} /$ day) intravenously in addition to symptomatic treatment. The patient's cough and expectoration were improving, and his temperature dropped to $37.4-38.8^{\circ} \mathrm{C}$. Upon admission, review of the patient's blood showed that the white blood cell count was $4.44 \times 10^{9} / 1$ (56.24\% neutrophils, $30 \%$ lymphocytes, and $13.37 \%$ monocytes). On the $25^{\text {th }}$ day of hospitalisation, a repeat CT scan revealed that the oval nodules were smaller and the cavity had essentially disappeared (Fig. 1B); moreover, his maxillofacial ulcers had become encrusted and the soft tissue swelling had subsided. On the $35^{\text {th }}$ day of hospitalisation, the patient refused further hospitalisation and continued taking voriconazole orally
$(0.4 \mathrm{~g} /$ day $)$ at home for two months. Two months later, a follow-up CT scan showed that the oval nodules were much smaller than in previous images (Fig. 1C). At the time of writing, the patient's temperature was almost normal, and a CT scan showed that the oval nodules had almost completely disappeared (Fig. 1D). The patient's nose remained deformed with no secretions, however.

\section{Discussion}

In the present case study, invasive pulmonary aspergillosis occurred in an agranulocytosis patient as a result of methimazole administration. Granulocytopaenia and agranulocytosis are common side effects of methimazole treatment. The incidence of granulocytopaenia is $3-12 \%$, while that of agranulocytosis is $0.1-0.6 \%$ [9], which is the one of the primary causes of death due to hyperthyroidism. Although hyperthyroidism is a cause of neutropaenia, agranulocytosis induced by hyperthyroidism is not common. Moreover, the patient's leukocytes were within normal limits before taking methimazole. After he started taking methimazole and accepted the treatment, his white blood cell count returned to normal. There was no leukopaenia during followed-up of at least two years. Consequently, we considered that his agranulocytosis was caused by the treatment with methimazole.

Aspergillus is an opportunistic pathogen, and immunocompromised patients who have experienced the administration of immunosuppressive therapy or glucocorticoids for more than three weeks, have had allogeneic hematopoietic stem cell or solid organ transplantation, or have a congenital immune deficiency are at risk of infection with invasive aspergillosis [10]. Recent evidence indicates that IPA can occur in patients without these factors; other relevant conditions include COPD, alcoholism, and severe sepsis [11-13].

Invasive pulmonary aspergillosis infections occur almost exclusively in immunocompromised patients [10]. Aspergillus fumigatus is the most common agent for Aspergillus infection in humans, accounting for $70-80 \%$ of these infections [14]. Aspergillus fumigatus spores are widely distributed in the environment. Most IPA is caused by inhaled Aspergillus spores, and the lower respiratory tract is the primary site of infection. Invasive pulmonary aspergillosis is seldom found on the skin, paranasal sinuses, gastrointestinal tract or other parts of the human body. This case also affected the paranasal sinus skin and lung tissue, which was likely caused when the skin infection spread to the lungs with the blood.

The definition of IPA in immunocompromised patients has recently been revised [10]. However, accurate diagnosis of IPA is difficult because the typical signs and symptoms are nonspecific. Culture and microscopic analysis of respiratory tract samples, such as sputum and bronchoalveolar lavage fluid (BALF), exhibit a sensitivity and spec- 
A

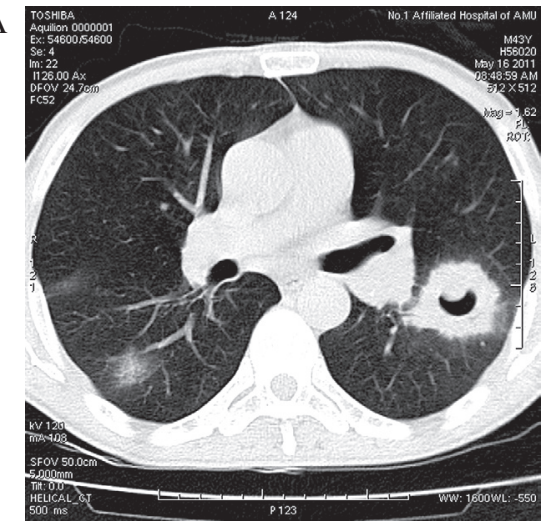

B

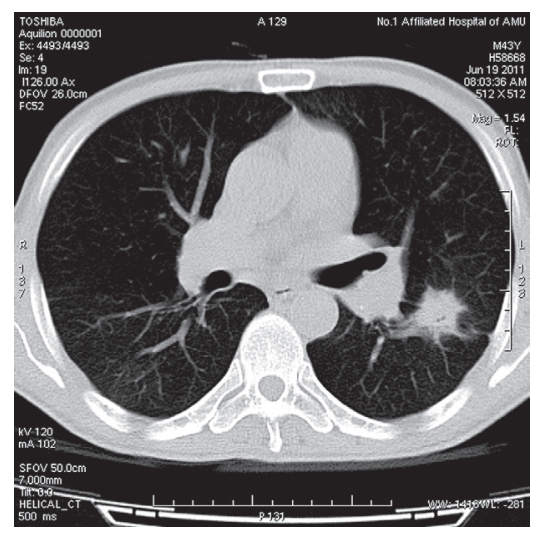

$\mathrm{C}$

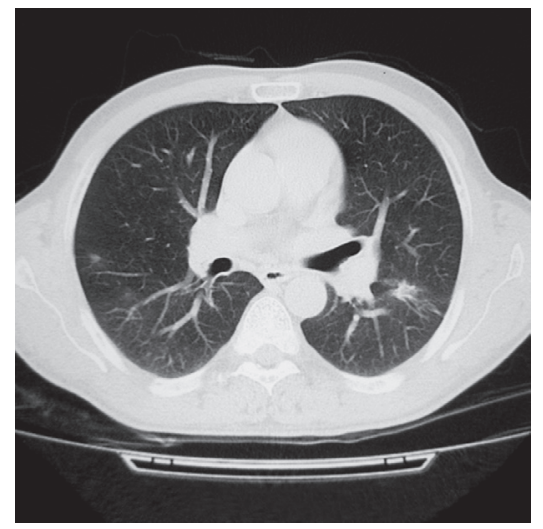

$\mathrm{D}$

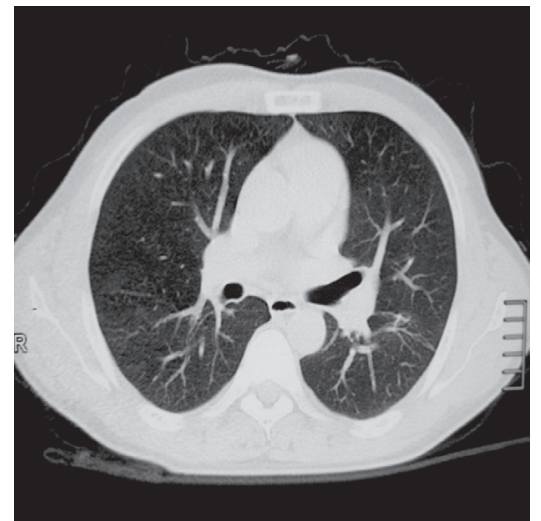

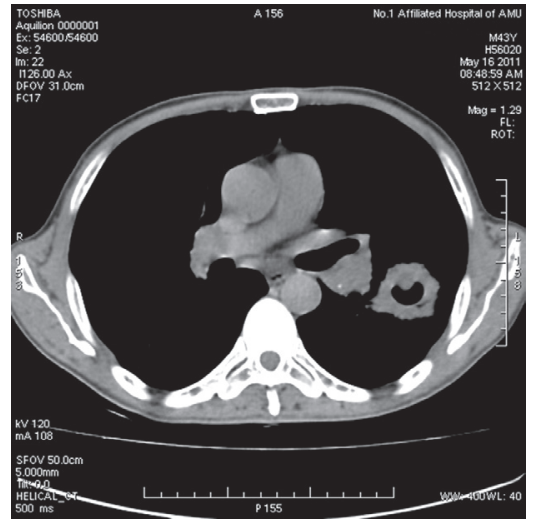
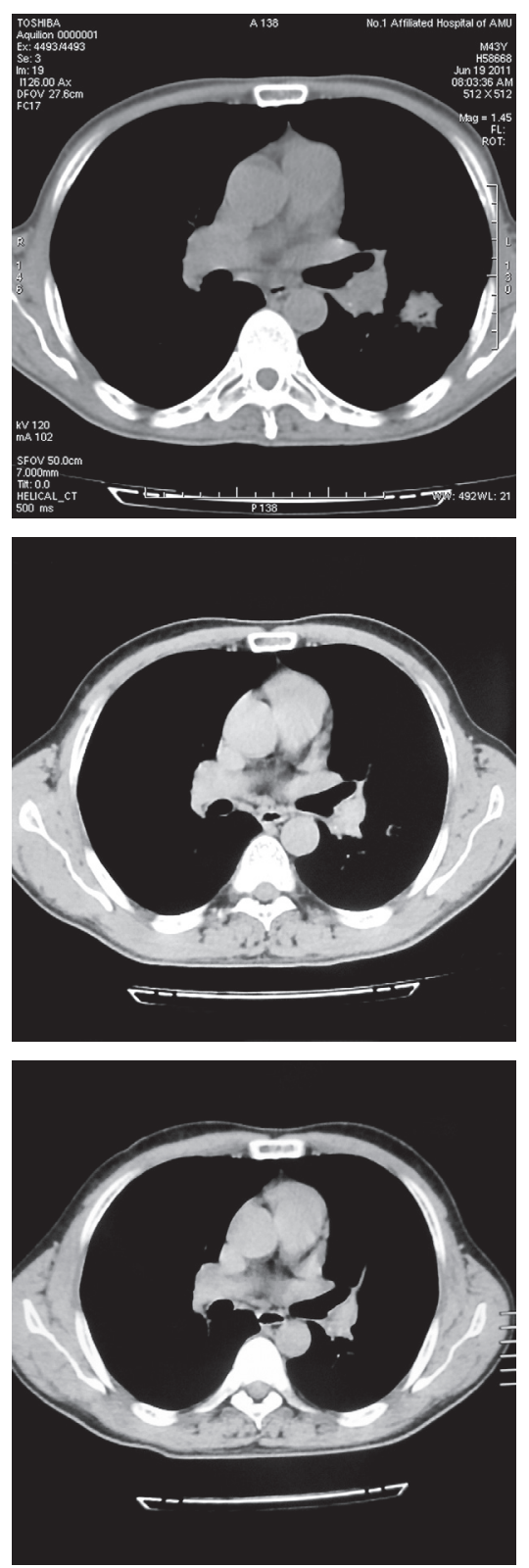

Fig. 1. A) In the initial chest CT, a 'halo sign' and 'air crescent sign' are visible. B) CT during hospitalization. C) CT after 2 months of treatment. D) CT after 11 months of treatment 

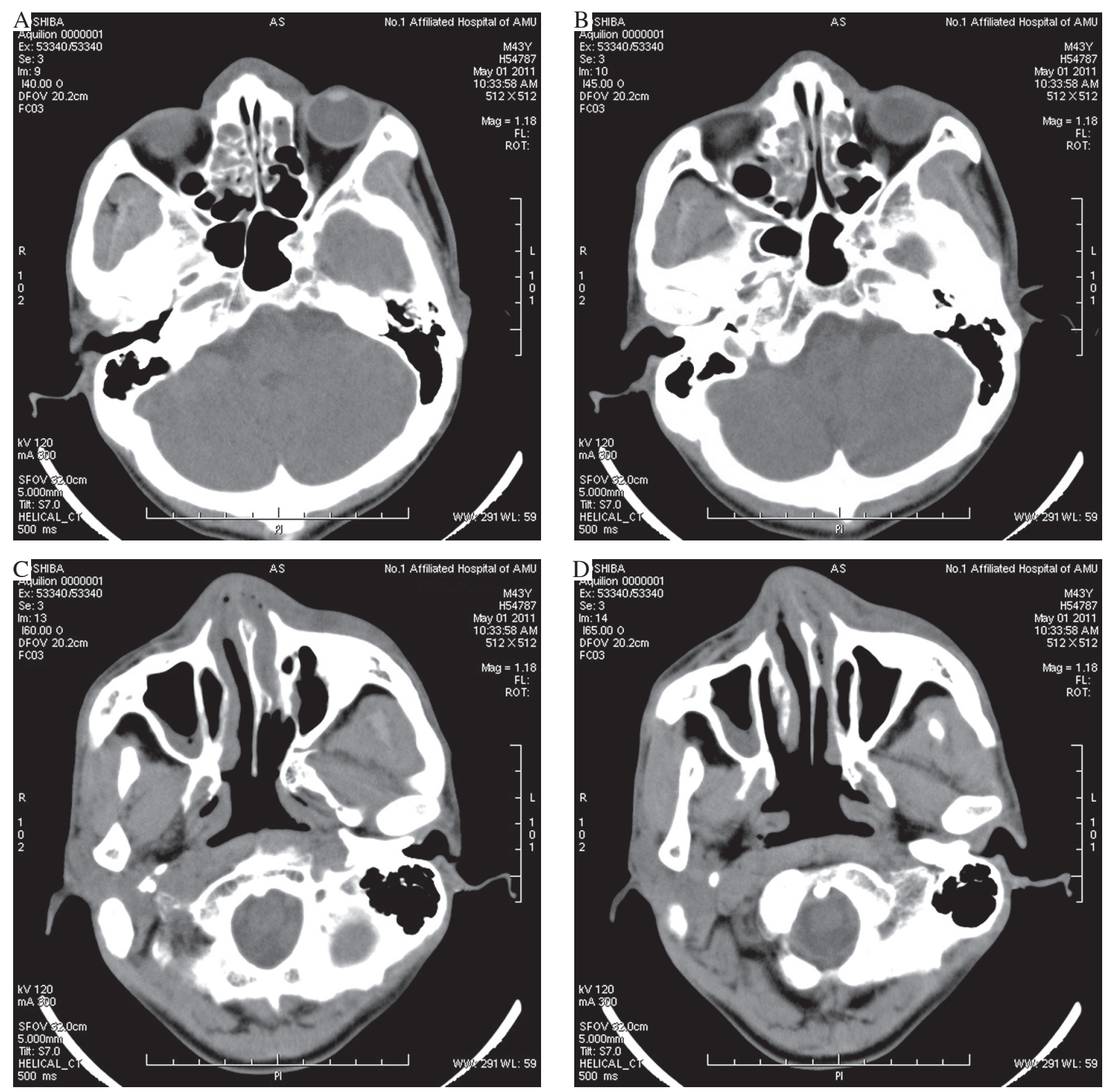

Fig. 2. A) Inflammation of the bilateral ethmoid sinus and B) maxillary sinus. C) Thickening of soft tissue on the left side of the ala nasi in the paranasal sinus CT. D) Maxillofacial soft tissue swelling

ificity of approximately 50\% [15]. A definitive diagnosis is obtained from tissue biopsies that demonstrate Aspergillus upon microscopic examination or in culture, and the endoscopic appearance of Aspergillus observed in this study is identical to observations from previous reports [16].

Fever, dyspnoea exacerbation, cough, chest pain, and haemoptysis are the most common complaints of patients presenting with IPA. In this case, the patient had a fever lasting for more than 20 days, accompanied by paroxysmal irritating cough and expectoration with white purulent sputum for two days, with a weight loss of $13 \mathrm{~kg}$, no chest pain, no haemoptysis, and no dyspnoea for the duration of disease. Computed tomography scanning and $(1,3)$ $\beta$-D-glucan have predictive value for early diagnosis [17], and the presence of $(1,3)-\beta$-D-glucan in the BALF is particularly useful [18]. In this case study, our patient's fungal infection was diagnosed through a combination of granulocytopaenia, serological tests (galactomannan antigen assay, $78.80 \mathrm{pg} / \mathrm{ml}$ ) and radiography tests, with a 'halo sign' and 'air crescent sign' in a CT being highly suggestive of IPA. Finally, microbiological histological data (A. fumigatus was detected in both fungal and bacterial cultures of sputum) confirmed the diagnosis of invasive aspergillosis infection. Vascular invasion by this fungus resulted in 

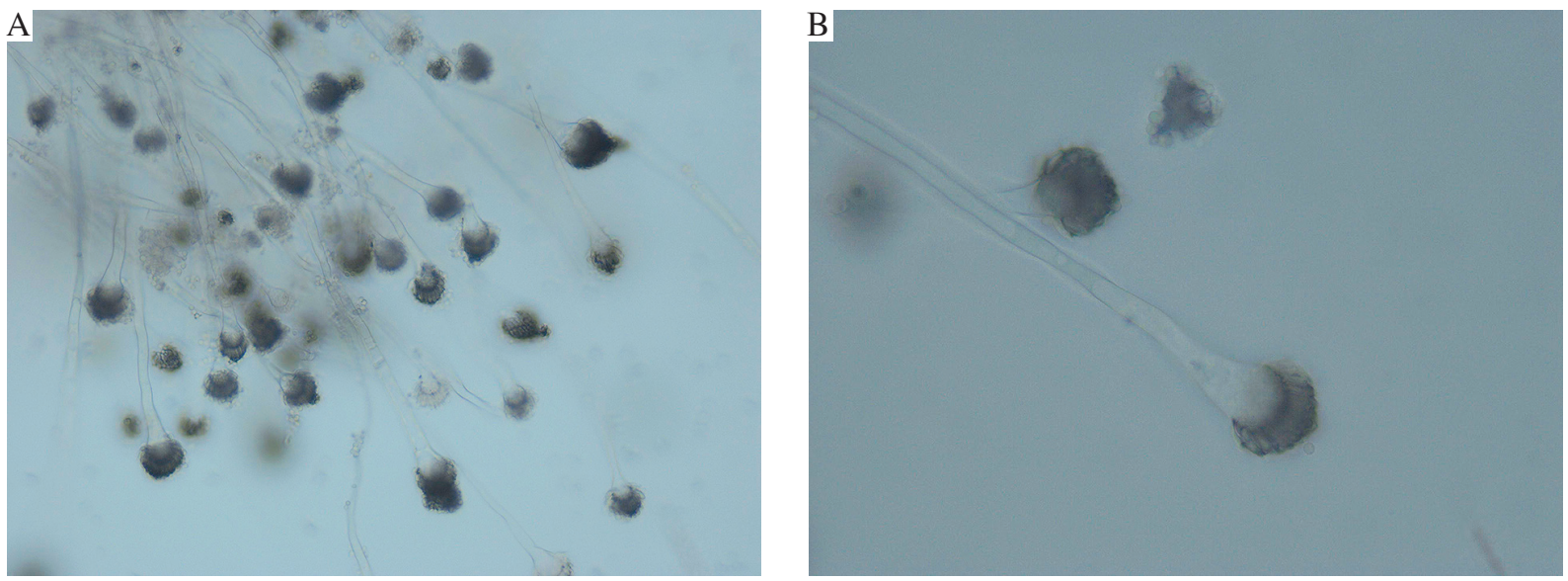

Fig. 3. Microscopic examination of Aspergillus fumigatus (A, 10× magnification), and microscopic examination revealing conidial heads (B, 40× magnification)

a thrombosis of small-to-medium-sized vessels that caused ischaemic necrosis.

As the clinical signs and symptoms of IPA patients are not typical, the diagnosis depends on tissue culture and histopathological examination; however, those patients who nurtured the same fungus by lower respiratory tract secretions repeatedly, with high risk factors for fungal infections as well as the typical imaging features, could have a clinical diagnosis made. Thereby, the patients with high risk factors of fungal infections should accept antifungal therapy as soon as possible if the anti-bacterial infection therapy is ineffective. Antifungal pharmacotherapy with voriconazole or amphotericin B can improve the chance of survival for patients with IPA [19]. Amphotericin B damages the kidney, however, so it is of limited use in the clinic. The antifungal medication triazole voriconazole is recommended as first-line treatment of IPA.

\section{References}

1. Varkey JB, Perfect JR (2008): Rare and emerging fungal pulmonary infections. Semin Respir Crit Care Med 29: 121-131.

2. Perkhofer S, Lass-Flörl C (2009): Anidulafungin and voriconazole in invasive fungal disease: pharmacological data and their use in combination. Expert Opin Investig Drugs 18: 1393-1404.

3. Lehrnbecher T, Frank C, Engels K, et al. (2010): Trends in the postmortem epidemiology of invasive fungal infections at a university hospital. J Infect 61: 259-265.

4. Liu YN, She DY, Sun TY, et al. (2011): A multicentre retrospective study of pulmonary mycosis clinically proven from 1998 to 2007. Zhonghua Jie He He Hu Xi Za Zhi 34: 86-90.

5. Chakrabarti A, Chatterjee SS, Das A, et al. (2011): Invasive aspergillosis in developing countries. Med Mycol 49: S35-47.

6. Niu YM, Tong ZH (2010): Invasive pulmonary aspergillosis in chronic obstructive pulmonary disease. Rev Med Chil 138: 612-620.

7. Bassetti M, Righi E, De Pascale G, et al. (2014): How to manage aspergillosis in non-neutropenic intensive care unit patients. Crit Care 18: 458.
8. Beck J, Broniszewska M, Schwienbacher M, et al. (2014): Characterization of the Aspergillus fumigatus chitosanase $\mathrm{CsnB}$ and evaluation of its potential use in serological diagnostics. Int J Med Microbiol 304: 696-702.

9. Ibáńez L, Vidal X, Ballarín E, et al. (2005): Population-based drug-induced agranulocytosis. Arch Intern Med 165: 869-874.

10. De Pauw B, Walsh TJ, Donnelly JP, et al. (2008): Revised definitions of invasive fungal disease from the European Organization for Research and Treatment of Cancer/Invasive Fungal Infections Cooperative Group and the National Institute of Allergy and Infectious Diseases Mycoses Study Group (EORTC/MSG) Consensus Group. Clin Infect Dis 46: 1813-1821.

11. Meersseman W, Lagrou K, Maertens J, et al. (2007): Invasive aspergillosis in the intensive care unit. Clin Infect Dis 45: 205-216.

12. Trof RJ, Beishuizen A, Debets-Ossenkopp YJ, et al. (2007): Management of invasive pulmonary aspergillosis in non-neutropenic critically ill patients. Intensive Care Med 33: 1694-1703.

13. Stevens DA, Melikian GL (2011): Aspergillosis in the "nonimmunocompromised' host. Immunol Invest 40: 751-766.

14. Barchiesi F, Mazzocato S, Mazzanti S, et al. (2014): Invasive aspergillosis in liver transplant recipients: Epidemiology, clinical characteristics, treatment and outcome in 116 cases. Liver Transpl 21: 204-212.

15. Ader F, Bienvenu AL, Rammaert B, Nseir S (2009): Management of invasive aspergillosis in patients with COPD: Rational use of voriconazole. Int J Chron Obstruct Pulmon Dis 4: 279-287.

16. Alhambra A, Catalán M, Moragues MD, et al. (2008): Isolation of Aspergillus lentulus in Spain from a critically ill patient with chronic obstructive pulmonary disease. Rev Iberoam Micol 25: 246-249.

17. Pfeiffer CD, Fine JP, Safdar N (2006): Diagnosis of invasive aspergillosis using a galactomannan assay: A meta-analysis. Clin Infect Dis 42: 1417-1427.

18. Meersseman W, Lagrou K, Maertens J, et al. (2008): Galactomannan in bronchoalveolar lavage fluid: a tool for diagnosing aspergillosis in intensive care unit patients. Am J Respir Crit Care Med 177: 27-34.

19. Takemoto K, Yamamoto Y, Ueda Y, et al. (2009): Comparative study on the efficacy of liposomal amphotericin B and voriconazole in a murine pulmonary aspergillosis model. Chemotherapy 55: 105-113. 